\title{
Detection of Acute Kidney Injury in Neonates after Cardiopulmonary Bypass
}

\author{
Tennille N. Webb \\ Pediatrics/Pediatric Nephrology, University of Alabama at Birmingham/Children's of Alabama, Birmingham, AL, USA
}

\section{Keywords}

Cardiac surgery-associated acute kidney injury •

Cardiopulmonary bypass · Kidney support therapy

\begin{abstract}
Cardiac surgery-associated acute kidney injury (CS-AKI) in neonates has been associated with poor outcomes. Early detection and intervention of acute kidney injury (AKI) are needed in order to mitigate some of these sequalae. Currently, serum creatinine ( $\mathrm{SCr}$ ) remains the gold standard for AKI diagnosis; however, changes are not seen until days after injury thus delaying the diagnosis. Serum creatinine in neonates varies based on multiple factors such as prematurity, the presence of maternal $\mathrm{SCr}$ and renal tubule immaturity. Acute kidney injury biomarkers, such as neutrophil gelatinase-associated lipocalin (NGAL), are useful for early AKI diagnosis. In addition to SCr and AKI biomarkers, a risk-based assessment of neonates at risk for CS-AKI could prove useful for early AKI diagnosis and intervention.
\end{abstract}

(c) 2022 S. Karger AG, Basel

Contribution from the AKI and CRRT 2021 Symposium at the 26th International Conference on Advances in Critical Care Nephrology, A Virtual/Hybrid Event from San Diego, CA, USA, February 28 to March 5,2021 . This symposium was supported in part by the NIDDK funded University of Alabama at Birmingham-University of California San Diego O'Brien Center for Acute Kidney Injury Research (P30DK079337).

Karger@karger.com

(c) 2022 S. Karger AG, Basel

www.karger.com/nef

Karger ${ }^{\prime}=$

\section{Introduction}

One well-known cause of acute kidney injury (AKI) is cardiac surgery-associated acute kidney injury (CS-AKI). There are significant differences between institutions in the incidence of CS-AKI, with estimates as high as $75 \%$ [1-6]. The Neonatal and Pediatric Heart and Renal Outcomes Network (NEPHRON) study is the first multicenter, retrospective neonatal cardiac study to describe the epidemiology of neonatal CS-AKI. The study included 22 hospitals and 2,040 neonates who underwent cardiac surgery and revealed CS-AKI in 54\% of the neonates [1]. Early diagnosis of CS-AKI is important in improving clinical outcomes. Once CS-AKI is diagnosed, interventions such as limiting nephrotoxin exposure, managing electrolytes and maintaining fluid balance can be implemented sooner. Early initiation of kidney support therapy (KST) is also an intervention that can be implemented once CS-AKI is diagnosed; however, is one that raises concern in these critically ill neonates. Apprehension regarding poor outcomes when performing KST on these critically ill neonates is not unfounded. Of some reassurance, previous studies have demonstrated that early initiation of KST in neonates requiring cardiac surgery has been associated with improved clinical outcomes including decreased mortality $[7,8]$. A retrospective analysis sought to determine if prophylactic peritoneal dialysis (PD) was beneficial in infants at high risk for CS-AKI after cardiopulmonary bypass (CPB). Prophylactic PD was 
found to be safe, negative fluid balance was achieved earlier and overall clinical outcomes were improved [9]. Additionally, the NEPHRON study demonstrated that prophylactic $\mathrm{PD}$ in neonates requiring $\mathrm{CPB}$ was not associated with CS-AKI [1].

Serum creatinine (SCr) is currently the gold standard for AKI detection; however, it is not the ideal biomarker because it is affected by other nonrenal factors including age, gender, muscle mass, fluid balance, and medications that may alter the tubular excretion of creatinine [10]. The AKI biomarker neutrophil gelatinase-associated lipocalin (NGAL) is a marker of tubular injury and is one of the most extensively studied biomarkers for AKI. Neutrophil gelatinase-associated lipocalin has been shown to be an early predictive marker of CS-AKI when measured $2 \mathrm{~h}$ after the initiation of CPB [11]. While NGAL has proven useful in early CS-AKI detection, there are clinical conditions in which NGAL is elevated aside from AKI including sepsis and urinary tract infections [12, 13]. Also, the availability of NGAL for generalized use as a biomarker for CS-AKI is limited among most institutions.

It remains a challenge to define AKI in neonates, as mentioned above, and it becomes more complex in neonates undergoing cardiac surgery because of their medical complexity. An assessment of CS-AKI risk in neonates undergoing cardiac surgery could be improved with the development of a risk stratification model. Renal angina and the renal angina index (RAI) are examples of the proposed model and have been validated in the pediatric population [14]. The objective of this review is to provide further insight into risk factors for neonatal CS-AKI after $\mathrm{CPB}, \mathrm{CS}-\mathrm{AKI}$ diagnosis and the potential use of a risk stratification model for detection and management of severe CS-AKI.

\section{Risk Factors for CS-AKI}

Understanding the short- and long-term risks of neonatal CS-AKI emphasizes the importance of identifying the associated risk factors. The ability to preemptively identify neonates who are likely to develop CS-AKI after CPB not only allows for early AKI detection but also the ability to formulate an intervention strategy in advance [15]. Such intervention strategies include appropriate dose adjustments of nephrotoxic medications that may be required for patient therapy (i.e., antibiotics), attentiveness to fluid balance including the ability to provide adequate hydration and nutrition to a neonate who may be oliguric/anuric, the possible need for prophylactic PD and timely preparation for KST if needed. Perioperative characteristics and associated outcomes of neonates requiring cardiac surgery were evaluated in the NEPHRON study. Neonatal AKI was defined by the neonatal modification of Kidney Disease: Improving Global Outcomes (KDIGO) criteria. Cardiac surgery-associated acute kidney injury developed in $59 \%$ of neonates who required $\mathrm{CPB}$ and in $38 \%$ who did not require CPB [1]. Demographic and perioperative risk factors associated with CSAKI were evaluated. Preoperative characteristics associated with CS-AKI included prostaglandin infusion, lowest preoperative $\mathrm{SCr}$, single ventricle physiology, need for $\mathrm{CPB}$, longer aortic cross clamp time, deep hypothermic cardiac arrest, surgery type, Society of Thoracic SurgeonsEuropean Association for Cardio-Thoracic Surgery (STAT) score, and urine output in the operating room. Multivariate analysis revealed that the duration of $\mathrm{CPB}$ (third tertile), use of modified ultrafiltration, and prophylactic PD were associated with CS-AKI. Delayed sternal closure and preoperative feeding were associated with lower CS-AKI. A subgroup multivariable analysis was performed only in neonates who required $\mathrm{CPB}$ and found that the duration of $\mathrm{CPB}$, modified ultrafiltration, and prophylactic PD were not associated with CS-AKI. Delayed sternal closure and preoperative feeding were also associated with lower CS-AKI in this group [1]. Previously described risk factors associated with CS-AKI such as longer duration of $\mathrm{CPB}$ were not found in the NEPHRON study. This difference may be secondary to the more homogenous neonatal cardiac cohort thus eliminating other potential confounders that may be present in other less homogenous studies.

\section{Is Serum Creatinine Enough?}

Despite its limitations, $\mathrm{SCr}$ is the most widely used marker of kidney injury likely due to its availability and low cost. Utilizing SCr to define AKI in neonates is cumbersome because of confounders including neonatal prematurity, the presence of maternal SCr and proximal tubular immaturity [16-18]. Neonatal SCr can take anywhere from a few days to weeks to reach its nadir as it is initially reflective of maternal SCr. Consequently, SCr changes seen within the first weeks of life may be considered normal. The SCr level in neonates who undergo cardiac surgery within the first week of life has not yet reached equilibrium, complicating the ability to detect AKI based solely on SCr. Serum creatinine is also affected by fluid status, and it has been demonstrated that failing to correct $\mathrm{SCr}$ for fluid overload underestimates the incidence of AKI [19]. More recently, neonatal AKI has been defined by the neonatal modification of KDIGO criteria; 
however, its use in a large neonatal cohort requiring cardiac surgery has not been evaluated $[1,20,21]$. The NEPHRON study used the neonatal modified KDIGO criteria to define CS-AKI and found that approximately $39 \%$ of neonates with CS-AKI had oliguria/anuria with normal SCr and approximately 35\% had elevated SCr with normal urine output. Only $26 \%$ of neonates met both the SCr and urine output criteria for CS-AKI diagnosis [1]. These findings further support the premise that use of SCr alone fails to identify a significant number of neonates that may have CS-AKI. Based on these findings, it would be safe to assume that indeed, SCr is not enough.

\section{Developing a Risk Stratification Model}

Development of a CS-AKI risk stratification model can be useful in assessing neonates at high risk for severe CS-AKI. An example of one such model is the renal angina index (RAI) $[14,22]$. The premise of renal angina was developed to objectively evaluate AKI risks comparable to the components of angina pectoris [23]. Renal angina identifies those at high risk of developing AKI and guides the use of AKI biomarker assessment for further investigation. The RAI uses a combination of risk factors (i.e., ICU admission, solid organ or stem cell transplantation) and early indications of renal injury (i.e., percent fluid accumulation, change in SCr) to identify individuals at higher risk for severe $\mathrm{AKI}$ and has been validated in the pediatric critical care population $[14,22,23]$. The RAI is a product of the assigned AKI risk and renal injury scores with a score of $>/=8$ fulfilling the definition of renal angina. A similar concept for neonates requiring cardiac surgery can be developed. Risk factors for neonates requiring cardiac surgery could include surgical complexity utilizing the STAT score, cardiac lesion such as single ventricle physiology and need for $\mathrm{CPB}$. As used in the current RAI model, changes in SCr and percent fluid accumulation would be used as early indicators of renal injury. The resulting score can then be utilized to determine which neonates will benefit from additional testing, such as NGAL or a furosemide challenge for further investigation and management of CS-AKI. As in the pediatric validated RAI, early interventions would be implemented in neonates with fulfillment of neonatal cardiac surgery-associated renal angina such as limiting nephrotoxin exposure and consideration for KST.

\section{Conclusion}

Cardiac surgery-associated AKI occurs commonly with the incidence varying significantly between institutions. Previously described risk factors once thought to be associated with CS-AKI were not found in a large multicenter neonatal cardiac study. Use of SCr alone is not sufficient in diagnosing CS-AKI. The AKI biomarker NGAL has proven to be useful in early CS-AKI detection; however, its limited availability makes it difficult to incorporate. Development of a risk stratification model specifically for neonates undergoing cardiac surgery would be beneficial for diagnoses and management of neonates at risk for severe CS-AKI.

\section{Conflict of Interest Statement}

The author has no conflicts of interest to declare.

\section{Funding Sources}

There were no funding sources for this work.

\section{References}

1 Alten JA, Cooper DS, Blinder JJ, Selewski DT, Tabbutt S, Sasaki J, et al. Epidemiology of acute kidney injury after neonatal cardiac surgery: a report from the multicenter neonatal and pediatric heart and renal outcomes network. Crit Care Med. 2021;49(10):e941-51.

2 Morgan CJ, Zappitelli M, Robertson CM, Alton GY, Sauve RS, Joffe AR, et al. Risk factors for and outcomes of acute kidney injury in neonates undergoing complex cardiac surgery. J Pediatr. 2013;162(1):120-7.e1.

3 Aydin SI, Seiden HS, Blaufox AD, Parnell VA, Choudhury T, Punnoose A, et al. Acute kidney injury after surgery for congenital heart disease. Ann Thorac Surg. 2012;94(5):1589-95.
4 Blinder JJ, Goldstein SL, Lee VV, Baycroft A, Fraser CD, Nelson D, et al. Congenital heart surgery in infants: effects of acute kidney injury on outcomes. J Thorac Cardiovasc Surg. 2012;143(2):368-74.

5 Blinder JJ, Asaro LA, Wypij D, Selewski DT, Agus MSD, Gaies M, et al. Acute kidney injury after pediatric cardiac surgery: a secondary analysis of the safe pediatric euglycemia after cardiac surgery trial. Pediatr Crit Care Med. 2017;18(7):638-46.

6 Kumar TK, Allen Ccp J, Spentzas MT, Berrios Ccp L, Shah MS, Joshi MVM, et al. Acute kidney injury following cardiac surgery in neonates and young infants: experience of a single center using novel perioperative strategies. World J Pediatr Congenit Heart Surg. 2016; 7(4):460-6.

7 Bojan M, Gioanni S, Vouhé PR, Journois D, Pouard P. Early initiation of peritoneal dialysis in neonates and infants with acute kidney injury following cardiac surgery is associated with a significant decrease in mortality. Kidney Int. 2012;82(4):474-81.

8 Sanchez-de-Toledo J, Perez-Ortiz A, Gil L, Baust T, Linés-Palazón M, Perez-Hoyos S, et al. Early initiation of renal replacement therapy in pediatric heart surgery is associated with lower mortality. Pediatr Cardiol. 2016; $37(4): 623-8$. 
9 Kwiatkowski DM, Menon S, Krawczeski CD, Goldstein SL, Morales DL, Phillips A, et al. Improved outcomes with peritoneal dialysis catheter placement after cardiopulmonary bypass in infants. J Thorac Cardiovasc Surg. 2015;149(1):230-6.

10 Edelstein CL. Biomarkers of acute kidney injury. Adv Chronic Kidney Dis. 2008;15(3): 222-34.

11 Krawczeski CD, Woo JG, Wang Y, Bennett MR, Ma Q, Devarajan P. Neutrophil gelatinase-associated lipocalin concentrations predict development of acute kidney injury in neonates and children after cardiopulmonary bypass. J Pediatr. 2011;158(6):1009-15.e1.

12 Patel ML, Sachan R, Shyam R, Kumar S, Kamal R, Misra A. Diagnostic accuracy of urinary neutrophil gelatinase-associated lipocalin in patients with septic acute kidney injury. Int J Nephrol Renovasc Dis. 2016;9:161-9.

13 Forster CS, Jackson E, Ma Q, Bennett M, Shah SS, Goldstein SL. Predictive ability of NGAL in identifying urinary tract infection in children with neurogenic bladders. Pediatr Nephrol. 2018;33(8):1365-74.
14 Basu RK, Zappitelli M, Brunner L, Wang Y, Wong HR, Chawla LS, et al. Derivation and validation of the renal angina index to improve the prediction of acute kidney injury in critically ill children. Kidney Int. 2014;85(3): 659-67.

15 Nada A, Bonachea EM, Askenazi DJ. Acute kidney injury in the fetus and neonate. Semin Fetal Neonatal Med. 2017;22(2):90-7.

16 Charlton JR, Boohaker L, Askenazi D, Brophy $\mathrm{PD}, \mathrm{D}$ 'Angio $\mathrm{C}$, Fuloria $\mathrm{M}$, et al. Incidence and Risk Factors of Early Onset Neonatal AKI. Clin J Am Soc Nephrol. 2019;14(2):184-95.

17 Weintraub AS, Carey A, Connors J, Blanco V, Green RS. Relationship of maternal creatinine to first neonatal creatinine in infants. J Perinatol. 2015;35(6):401-4.

18 Gallo D, de Bijl-Marcus KA, Alderliesten T, Lilien M, Groenendaal F. Early acute kidney injury in preterm and term neonates: incidence, outcome, and associated clinical features. Neonatology. 2021;118(2):174-9.

19 Basu RK, Andrews A, Krawczeski C, Manning P, Wheeler DS, Goldstein SL. Acute kidney injury based on corrected serum creatinine is associated with increased morbidity in children following the arterial switch operation. Pediatr Crit Care Med. 2013;14(5):e218-24.

20 Zappitelli M, Ambalavanan N, Askenazi DJ, Moxey-Mims MM, Kimmel PL, Star RA, et al. Developing a neonatal acute kidney injury research definition: a report from the NIDDK neonatal AKI workshop. Pediatr Res. 2017; 82(4):569-73.

21 Jetton JG, Boohaker LJ, Sethi SK, Wazir S, Rohatgi S, Soranno DE, et al. Incidence and outcomes of neonatal acute kidney injury (AWAKEN): a multicentre, multinational, observational cohort study. Lancet Child Adolesc Health. 2017;1(3):184-94.

22 Basu RK, Kaddourah A, Goldstein SL. Assessment of a renal angina index for prediction of severe acute kidney injury in critically ill children: a multicentre, multinational, prospective observational study. Lancet Child Adolesc Health. 2018;2(2):112-20.

23 Chawla LS, Goldstein SL, Kellum JA, Ronco C. Renal angina: concept and development of pretest probability assessment in acute kidney injury. Crit Care. 2015;19:93. 